\title{
A Statistically Efficient Estimator for Co-array Based DoA Estimation
}

\author{
Saeid Sedighi, Bhavani Shankar M. R. and Björn Ottersten \\ Interdisciplinary Centre for Security, Reliability and Trust (SnT), University of Luxembourg, Luxembourg \\ Emails: \{saeid.sedighi,bhavani.shankar,bjorn.ottersten\}@uni.lu
}

\begin{abstract}
Co-array-based Direction of Arrival (DoA) estimation using Sparse linear arrays (SLAs) has recently gained considerable interest in array processing due to the attractive capability of providing enhanced degrees of freedom. Although a variety of estimators have been suggested in the literature for co-array-based DoA estimation, none of them are statistically efficient. This work introduces a novel Weighted Least Squares (WLS) estimator for the co-array-based DoA estimation employing the covariance fitting method. Then, an optimal weighting is given so that the asymptotic performance of the proposed WLS estimator coincides with the Cramér-Rao Bound (CRB), thereby ensuring statistical efficiency of resulting WLS estimator. This implies that the proposed WLS estimator has significantly better performance compared to existing methods in the literature. Numerical simulations are provided to corroborate the asymptotic statistical efficiency and the improved performance of the proposed estimator.
\end{abstract}

\section{INTRODUCTION}

It is widely known that ULAs are not capable of identifying more sources than the number of physical elements in the array [1], [2]. To transcend this limitation, exploitation of Sparse Linear Arrays (SLAs) with particular geometries, such as Minimum Redundancy Arrays (MRAs) [3], co-prime arrays [4] and nested arrays [5] has been proposed. These arrays can dramatically boost the degrees of freedom by employing the difference co-array model such that significantly more sources than the number of physical elements in the array can be identified. This attractive feature has spurred further research in co-array-based DoA estimation using SLAs in recent years.

Recently, a detailed study on co-array-based DoA estimation through an analysis of the Cramér-Rao Bound (CRB) has been conducted in [6]. The findings in [6] provide valuable insights into the performance limits of co-array-based DoA estimation, which are useful for benchmarking. Further, a number of DoA estimators based on the co-array model have been proposed in the literature. In [5], [7], [8], DoAs are estimated by applying conventional subspace methods such as MUSIC, ESPRIT on an Augmented Sample Covariance Matrix (ASCM) obtained from the original sample covariance matrix. There are two different ways for constructing the ASCM, namely, 1) the direct augmentation approach [9]; 2) spatial smoothing approach [5]. It is shown that applying MUSIC on both leads to the same asymptotic Mean Square Error (MSE), but the former is computationally efficient. Some sparsity-based methods are also given in [10]-[12] by imposing sparsity constraints on source profiles. Recently, the current authors have also proposed the use of a Least Squares estimator [13].
However, performance of these proposed estimators does not approach the CRB, leading them to be statistically inefficient.

In this paper, we propose a new Weighted Least Squared (WLS) estimator for the co-array based DoA estimation using covariance fitting method. It is shown that by selecting the weighting matrix optimally, the error covariance matrix of DoA estimates coincides with the CRB, resulting in an asymptotic statistically efficient estimator. Further, some simulation results are provided to compare the performance of the proposed WLS estimator with the estimators proposed in the literature.

Notation: Vectors and matrices are referred to by lowerand upper-case bold-face, respectively. The superscripts $*, T$, $H$ denote the conjugate, transpose and Hermitian (conjugate transpose) operations, respectively. $\|\mathbf{a}\|_{2}$ stands for the $\ell_{2}$ norm of $\mathbf{A}$ and $\mathbf{a} .[\mathbf{A}]_{i, j}$ and $[\mathbf{a}]_{i}$ indicate the $(i, j)^{\text {th }}$ and $i^{\text {th }}$ entry of $\mathbf{A}$ and $\mathbf{a}$, respectively. $\hat{\mathbf{A}}$ and $\hat{\mathbf{a}}$ denote the estimate of $\mathbf{A}$ and $\mathbf{a}$, respectively. $\left(a_{1}, a_{2}, \cdots, a_{n}\right)$ is an $n$-tuple with elements of $a_{1}, a_{2}, \cdots, a_{n} .|\mathbb{A}|$ represents the cardinality of the set $\mathbb{A}$. $\operatorname{diag}(\mathbf{a})$ is a diagonal matrix whose diagonal entries are equal to the elements of a. The $M \times M$ identity matrix is denoted by $\mathbf{I}_{M} \cdot \mathbb{E}\{$.$\} stands for the statistical expecta-$ tion. $\otimes$ and $\odot$ represent Kronecker and Khatri-Rao products, respectively. $\operatorname{Vec}(\mathbf{A})=\left[\begin{array}{llll}\mathbf{a}_{1}^{T} & \mathbf{a}_{2}^{T} & \cdots & \mathbf{a}_{n}^{T}\end{array}\right]^{T}$ denotes the vectorization operation. $\mathbf{A}^{\dagger}$ and $\Pi_{\mathbf{A}}^{\perp}$ indicate the pseudoinverse and the projection matrix onto the null space of the full column rank matrix $\mathbf{A}$, respectively.

\section{Co-ARray System Model}

We consider an SLA with $M$ elements located at positions $\left(m_{1} \frac{\lambda}{2}, m_{2} \frac{\lambda}{2}, \cdots, m_{M} \frac{\lambda}{2}\right)$ with $m_{i} \in \mathrm{M}$. Here $\mathbb{M}$ is an integer set with cardinality $|\mathrm{M}|=M$, and $\lambda$ represents the wavelength of the incoming signals. It is assumed $K$ narrowband signals with distinct DoAs $\boldsymbol{\theta}=\left[\begin{array}{llll}\theta_{1} & \theta_{2} & \cdots & \theta_{K}\end{array}\right]^{T}$ impinge on the SLA from far field. Accordingly, the vector of signals received by the SLA at time instance $t$ can be modeled as

$$
\mathbf{y}(t)=\mathbf{A}(\boldsymbol{\theta}) \mathbf{x}(t)+\mathbf{n}(t) \in \mathbb{C}^{M \times 1}, \quad t=1, \cdots, N,
$$

where $\mathbf{x}(t) \in \mathbb{C}^{K \times 1}$ denotes the vector of source signals, $\mathbf{n}(t) \in \mathbb{C}^{M \times 1}$ is additive noise, and $\mathbf{A}(\boldsymbol{\theta})=$ $\left[\mathbf{a}\left(\theta_{1}\right), \quad \mathbf{a}\left(\theta_{2}\right), \cdots \mathbf{a}\left(\theta_{K}\right)\right] \in \mathbb{C}^{M \times K}$ represents the SLA steering matrix where

$$
\mathbf{a}\left(\theta_{i}\right)=\left[\begin{array}{llll}
e^{\mathrm{j} \pi \sin \theta_{i} m_{1}} & e^{\mathrm{j} \pi \sin \theta_{i} m_{2}} & \cdots & e^{\mathrm{j} \pi \sin \theta_{i} m_{M}}
\end{array}\right]^{T},
$$


is the SLA manifold vector for the $i^{\text {th }}$ signal. Further, the following assumptions are made on source signals and noise:

A1 The noise vector follows a zero-mean circular complex Gaussian distribution with the covariance matrix, $\mathbb{E}\left\{\mathbf{n}(t) \mathbf{n}^{H}(t)\right\}=\sigma^{2} \mathbf{I}_{M}$.

A2 The source signal vector is modeled as a zero-mean circular complex Gaussian random vector with covariance matrix $\mathbb{E}\left\{\mathbf{x}(t) \mathbf{x}^{H}(t)\right\}=\operatorname{diag}(\mathbf{p})$ where $\mathbf{p}=$ $\left[\begin{array}{llll}p_{1}, & p_{2}, & \cdots & p_{K}\end{array}\right]^{T} \in \mathbb{R}_{>0}^{K \times 1}$ (i. e., $p_{l}>0, \forall l$ ).

A3 Source signal and noise vectors are mutually independent.

A4 There is no temporal correlation between the snapshots, i.e., $\mathbb{E}\left\{\mathbf{n}\left(t_{1}\right) \mathbf{n}^{H}\left(t_{2}\right)\right\}=\mathbb{E}\left\{\mathbf{x}\left(t_{1}\right) \mathbf{x}^{H}\left(t_{2}\right)\right\}=\mathbf{0}$ when $t_{1} \neq$ $t_{2}$ and $\mathbf{0}$ is an all zero matrix of appropriate dimensions.

Based on the above assumptions, the covariance matrix of the received signals, i.e., $\mathbf{R}=\mathbb{E}\left\{\mathbf{y}(t) \mathbf{y}^{H}(t)\right\}$, is given by

$$
\mathbf{R}=\mathbf{A}(\boldsymbol{\theta}) \operatorname{diag}(\mathbf{p}) \mathbf{A}^{H}(\boldsymbol{\theta})+\sigma^{2} \mathbf{I}_{M} \in \mathbb{C}^{M \times M} .
$$

Following [5]-[7], the difference co-array model of the SLA is obtained by vectorizing the covariance matrix in (3), which results in

$$
\begin{aligned}
\mathbf{r} & \doteq \operatorname{Vec}(\mathbf{R})=\left(\mathbf{A}^{*}(\boldsymbol{\theta}) \odot \mathbf{A}(\boldsymbol{\theta})\right) \mathbf{p}+\sigma^{2} \operatorname{Vec}\left(\mathbf{I}_{M}\right), \\
& =\mathbf{J A}_{d}(\boldsymbol{\theta}) \mathbf{p}+\sigma^{2} \mathbf{J} \mathbf{g} \in \mathbb{C}^{M^{2} \times 1},
\end{aligned}
$$

where $\mathbf{A}_{d}(\boldsymbol{\theta}) \in \mathbb{C}^{(2 D-1) \times K}$ corresponds to the steering matrix of the difference co-array whose elements are located at $\left(-\ell_{D-1} \frac{\lambda}{2}, \cdots, 0, \cdots, \ell_{D-1} \frac{\lambda}{2}\right)$ with $\ell_{i} \in \mathbb{D}=\left\{\mid m_{p}-\right.$ $\left.m_{q}|| m_{p}, m_{q} \in \mathbb{M}\right\}$ and $D=|\mathbb{D}|$. Further, $\mathbf{g} \in\{0,1\}^{(2 D-1) \times 1}$ is a column vector with $[\mathrm{g}]_{i}=\delta[i-D]$, and the selection matrix $\mathbf{J}$ is represented as follows

Definition 1. The binary matrix $\mathbf{J} \in\{0,1\}^{M^{2} \times(2 D-1)}$ is defined as [6]

$\mathbf{J}=\left[\begin{array}{lllll}\operatorname{Vec}\left(\mathbf{L}_{D-1}^{T}\right) & \cdots & \operatorname{Vec}\left(\mathbf{L}_{0}\right) & \cdots & \operatorname{Vec}\left(\mathbf{L}_{D-1}\right),\end{array}\right]$, where

$\left[\mathbf{L}_{n}\right]_{p, q}=\left\{\begin{array}{lc}1, & \text { if } m_{p}-m_{q}=\ell_{n}, \\ 0, & \text { otherwise, }\end{array}\right.$

with $1 \leq p, q \leq M$ and $0 \leq n \leq D-1$.

The difference co-array model in (4) can be perceived to be the response of a virtual array whose steering matrix is given by $\mathbf{A}_{d}(\boldsymbol{\theta})$ to the parameter vector with signal powers $\mathbf{p}$ in presence of the noise vector $\sigma^{2} \operatorname{Vec}\left(\mathbf{I}_{M}\right)$. This virtual array includes a contiguous ULA segment around the origin with the size of $2 v-1$ where $v$ is the largest integer such that $\{0,1, \cdots, v-1\} \subseteq \mathbb{D}$. It has been shown in [4]-[6] that the size of the contiguous ULA segment of the difference co-array plays a crucial role in the number of identifiable sources such that $K$ distinct sources are identifiable if $K \leq v-1$. Hence, in case the SLA is designed properly such that $v>M$, we are able to identify more sources than the number of physical elements in the SLA, exploiting the source signal covariance matrix structure efficiently. An illustrative example of an SLA and its co-array is provided in Fig. 1. (a)

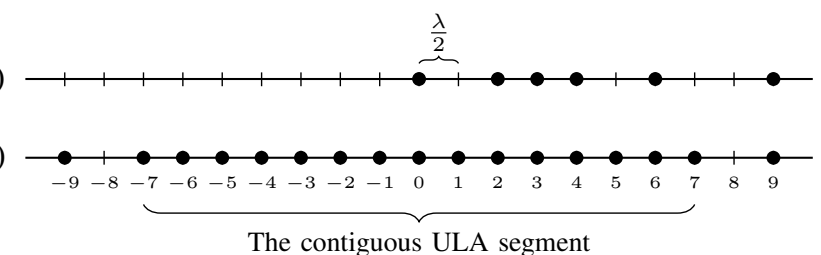

Fig. 1. Array geometry of a co-prime array with 6 elements: (a) physical array with $\mathbb{M}=\{0,2,3,4,6,9\} ;$ (b) difference co-array with $\mathbb{D}=$ $\{0,1,2,3,4,5,6,7,9\}$.

\section{CO-ARRAY BASED WLS ESTIMATOR}

The problem under consideration is to estimate the unknown parameters - DoAs, source signal powers and the noise variance - in (4) using array observations, i.e., $\{\mathbf{y}(t)\}_{t=1}^{N}$. Of these, DoAs are of primary interest and the other parameters are of subordinate interest. However, the estimation of the secondary parameters is essential for accurate DoA estimation. Since estimation of $\sigma^{2}$ and $\mathbf{p}$ is not the main aim here, a consistent estimate of them suffices for our purpose. In what follows, we first introduce a consistent estimate of the noise variance and then formulate and present the WLS estimator for DoAs and source signal powers.

Let $\hat{\mathbf{R}}$ denote the sample covariance matrix, defined as

$$
\hat{\mathbf{R}}=\frac{1}{N} \sum_{t=1}^{N} \mathbf{y}(t) \mathbf{y}^{H}(t) \in \mathbb{C}^{M \times M},
$$

and $\hat{\mathbf{r}}=\operatorname{Vec}(\hat{\mathbf{R}})$ denote its vectorized form. In addition, let $\hat{\mathbf{R}}_{v}$ be the augmented sample covariance matrix, which is constructed as follows [9]

$$
\hat{\mathbf{R}}_{v}=\left[\begin{array}{llll}
\mathbf{T}_{v} \mathbf{J}^{\dagger} \hat{\mathbf{r}} & \mathbf{T}_{v-1} \mathbf{J}^{\dagger} \hat{\mathbf{r}} & \cdots & \mathbf{T}_{1} \mathbf{J}^{\dagger} \hat{\mathbf{r}}
\end{array}\right] \in \mathbb{C}^{v \times v},
$$

where $\mathbf{T}_{i}$ is a selection matrix defined as

$$
\mathbf{T}_{i}=\left[\begin{array}{lll}
\mathbf{0}_{v \times(i+D-v-1)} & \mathbf{I}_{v} & \mathbf{0}_{v \times(D-i)}
\end{array}\right] \in\{0,1\}^{v \times(2 D-1)}
$$

Then, a consistent estimate of the noise variance is given by

$$
\widehat{\sigma^{2}}=\frac{\operatorname{Vec}^{H}\left(\hat{\mathbf{U}}_{n} \hat{\mathbf{U}}_{n}^{H}\right) \mathbf{T} \mathbf{J}^{\dagger} \hat{\mathbf{r}}}{v-K}
$$

where $\hat{\mathbf{U}}_{n}$ represents the eigenvectors of the augmented sample covariance matrix $\hat{\mathbf{R}}_{v}$ corresponding to its $v-K$ smallest eigenvalues and $\mathbf{T}=\left[\begin{array}{llll}\mathbf{T}_{v}^{T} & \mathbf{T}_{v-1}^{T} & \cdots & \mathbf{T}_{1}^{T}\end{array}\right]^{T} \in$ $\mathbb{C}^{v^{2} \times(2 D-1)}$.

Now, to estimate source DoAs from (4), it is possible to formulate the co-array-based LS estimates of $\boldsymbol{\theta}, \mathbf{p}$ as

$$
\left[\begin{array}{c}
\hat{\boldsymbol{\theta}}_{l s} \\
\hat{\mathbf{p}}_{l s}
\end{array}\right]=\underset{\boldsymbol{\theta}, \mathbf{p}}{\operatorname{argmin}}\left\|\mathbf{r}-\mathbf{J} \mathbf{A}_{d}(\boldsymbol{\theta}) \mathbf{p}-\widehat{\sigma^{2}} \operatorname{Vec}\left(\mathbf{I}_{M}\right)\right\|_{2}^{2} .
$$

However, our investigations, presented in [13], indicate that the LS estimates of DoAs do not show a significant performance improvement in terms of MSE compared to the existing algorithms. Thus it would be useful to introduce a weighting 
in the above criterion to achieve better performance. Hence, we propose the following WLS estimator instead

$$
\left[\begin{array}{c}
\hat{\boldsymbol{\theta}}_{w l s} \\
\hat{\mathbf{p}}_{w l s}
\end{array}\right]=\underset{\boldsymbol{\theta}, \mathbf{p}}{\operatorname{argmin}}\left\|\mathbf{W}^{\frac{1}{2}}\left(\mathbf{r}-\mathbf{J} \mathbf{A}_{d}(\boldsymbol{\theta}) \mathbf{p}-\widehat{\sigma^{2}} \operatorname{Vec}\left(\mathbf{I}_{M}\right)\right)\right\|_{2}^{2} .
$$

where $\mathbf{W}$ is a positive definite weighting matrix. The weighting matrix $\mathbf{W}$ should be determined to minimize the MSE of DoA estimates. Inserting (10) into (12) and performing certain algebraic manipulations leads to the following estimates for source signal powers and DoAs

$$
\begin{aligned}
\hat{\mathbf{p}}_{w l s} & =\left(\mathbf{W}^{\frac{1}{2}} \mathbf{J} \mathbf{A}_{d}(\boldsymbol{\theta})\right)^{\dagger} \mathbf{W}^{\frac{1}{2}} \hat{\mathbf{Q}} \hat{\mathbf{r}} . \\
\hat{\boldsymbol{\theta}}_{w l s} & =\underset{\boldsymbol{\theta}}{\operatorname{argmin}}\left\|\Pi_{\mathbf{W}^{\frac{1}{2}} \mathbf{J A}_{d}(\boldsymbol{\theta})}^{\perp} \mathbf{W}^{\frac{1}{2}} \hat{\mathbf{Q}} \hat{\mathbf{r}}\right\|_{2}^{2} .
\end{aligned}
$$

where

$$
\hat{\mathbf{Q}} \doteq \mathbf{I}_{M^{2}}-\frac{\operatorname{Vec}\left(\mathbf{I}_{M}\right) \operatorname{Vec}^{H}\left(\hat{\mathbf{U}}_{n} \hat{\mathbf{U}}_{n}^{H}\right) \mathbf{T} \mathbf{J}^{\dagger}}{v-K}
$$

If the weighting matrix is selected as follows

$$
\mathbf{W}=\left(\Pi_{\mathbf{J A}_{d}(\boldsymbol{\theta})}^{\perp} \mathbf{S} \Pi_{\mathbf{J A}_{d}(\boldsymbol{\theta})}^{\perp}+\mathbf{J} \mathbf{A}_{d}(\boldsymbol{\theta}) \mathbf{A}_{d}^{H}(\boldsymbol{\theta}) \mathbf{J}^{T}\right)^{-1},
$$

where

$$
\mathbf{S}=\mathbf{Q}\left(\mathbf{R}^{T} \otimes \mathbf{R}\right) \mathbf{Q}^{H}+\mathbf{b b}^{H}
$$

and $\mathbf{b} \doteq \mathbf{J}^{\dagger H} \mathbf{T}^{H} \operatorname{Vec}\left(\mathbf{U}_{n} \mathbf{U}_{n}^{H}\right)$, then the asymptotic performance of the proposed WLS estimator in (14) achieves the CRB. The details of proof will be given in the journal extension of this paper [14].

Remark 1. We note that the optimal weighting matrix given in (16) depends on the true value of the parameters. However, in practice, it can be replaced with a consistent estimate without affecting the asymptotic performance of the WLS estimator [15]-[18]. To this end, we can first use any other consistent estimator like MUSIC or ESPRIT to obtain an initial estimate of $\boldsymbol{\theta}$. Then, we compute a consistent estimate of the optimal weighting matrix based on the initial estimate of $\boldsymbol{\theta}$. Finally, we use the WLS estimator given in (14) to derive statistically efficient estimates of DoAs.

\section{Simulation Results}

In this section, we provide some numerical results to show the statistical efficiency of the proposed WLS estimator and to compare its performance with those of the existing estimators in the literature. Specifically, we compare the performance of the WLS estimator proposed in this paper with that of coarray-based MUSIC [5], [7] and co-array-based ESPRIT [8] and illustrate the superior performance of the WLS estimator.

In all experiments, each simulated point has been computed by 5000 Monte Carlo repetitions. In addition, it is assumed that the sources are located at $\left\{-60^{\circ}+120^{\circ}(k-1) /(K-\right.$ 1) $\mid k=0,1, \cdots, K-1\}$ in case $K$ independent sources are available. All sources have equal powers, i.e., $p_{1}=p_{2}=\cdots=$ $p_{K}=p$, and the SNR is defined as $10 \log \frac{p}{\sigma^{2}}$. Throughout this

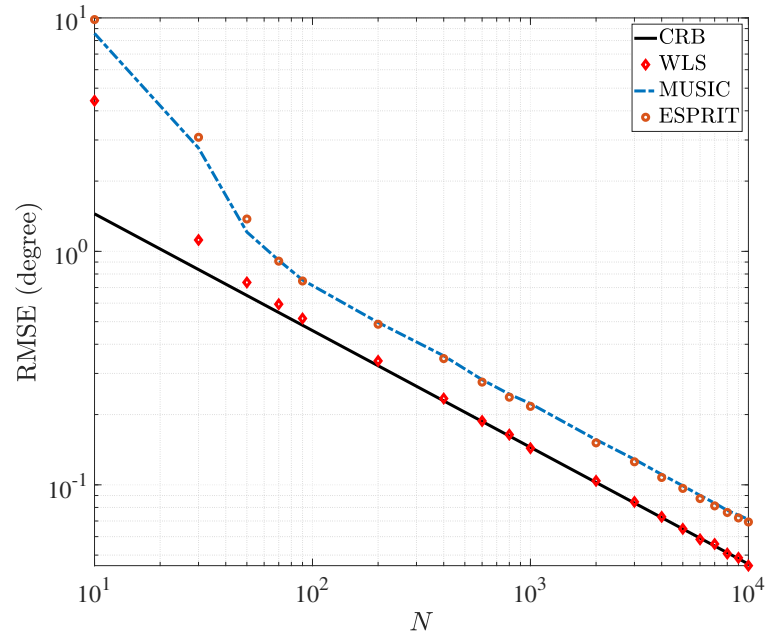

Fig. 2. RMSE in degree for $\theta_{2}$ versus SNR for a nested array with $M=6$ elements and configuration given in (18), $N=500$, and $K=7>M$.

section, we use a nested array with 6 physical elements and the following geometry:

$$
\mathrm{M}_{\text {nested }}:\{1,2,3,4,8,12\}
$$

This SLA generates a difference co-array described as follows:

$$
\mathbb{D}_{\text {nested }}:\{0,1,2,3,4,5,6,7,8,9,10,11\},
$$

Fig. 2 depicts the Root-Mean-Squares-Error (RMSE) for $\theta_{2}$ in degree versus the number of snapshots with the following setup. We consider a nested array with $M=6$ elements and configuration given in (18). The SNR is assumed to be $3 \mathrm{~dB}$ and $K=7>M$. Fig. 2 shows that there is a good agreement between the RMSE of the proposed WLS estimator and the CRB for the both scenarios when almost 100 snapshots are available. This implies that the proposed WLS estimator becomes statistically efficient even for finite number of samples (e.g., 100 in the scenario of Fig 2). Further, a considerable gap is observed between the performance of co-array-based MUSIC (ESPRIT) and the CRB, arising from their statistical inefficiency. When the number of snapshots is less than 100, all estimators are confronted with significant performance degradation. This happens because the sample covariance matrix is unable to provide an accurate estimate of $\mathbf{R}$ under such circumstances due to lack of snapshots. However, the proposed WLS estimator has even superior performance beside the existing estimators in this region.

Fig. 3 shows the RMSE for $\theta_{2}$ in degree versus SNR for the same setup used for Fig. 2. The number of snapshots is considered to be $N=500$. It is seen that the RMSE of the WLS estimator perfectly matches the CRB over the considered SNR range, arising from its statistical efficiency. In addition, it is observed that for $K=4<M$, similar to CRB, the RMSE of WLS estimator approaches zero as SNR increases while the RMSEs of co-array-based MUSIC and ESPRIT saturate at the SNR around $5 \mathrm{~dB}$. This means that while the WLS estimator remains statistically efficient for the whole range 
To appear in the proceedings of the $52^{\text {nd }}$ annual Asilomar Conference on Signals, Systems, and Computers, Oct. 2018.

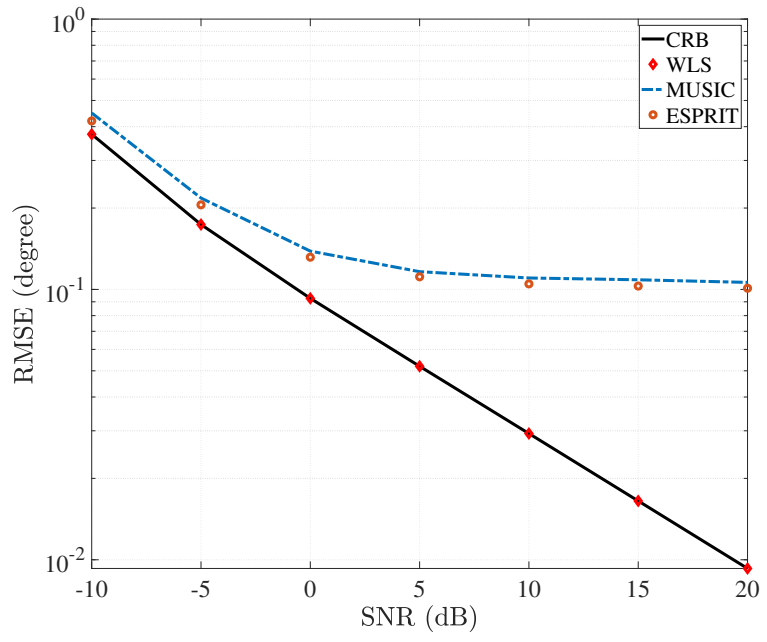

(a)

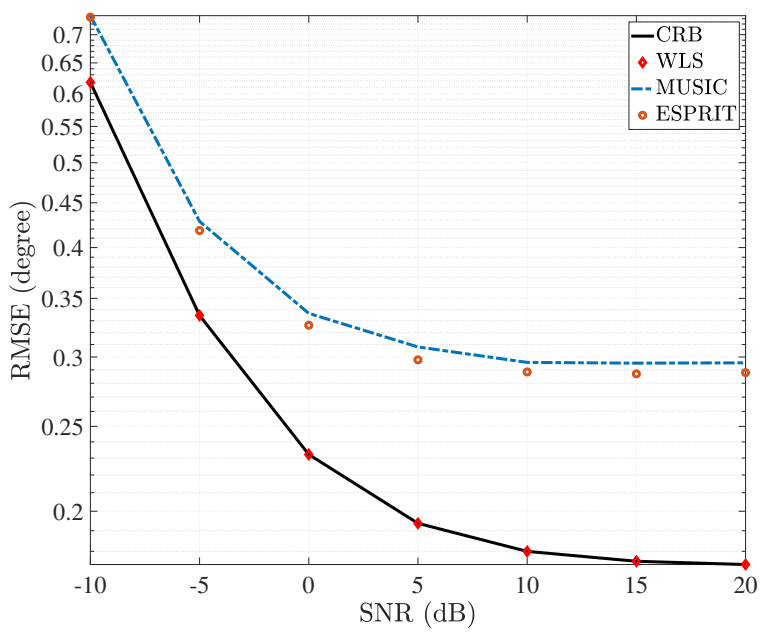

(b)

Fig. 3. RMSE in degree for $\theta_{2}$ versus SNR for a nested array with $M=6$ elements and configuration given in (18), $N=500$, and: (a) $K=4<M$; (b) $K=7>M$.

of the SNR, co-array-based MUSIC and ESPRIT become inefficient with the increase in SNR. When $K=7<M$, the RMSE of the WLS estimator as well as CRB get saturated at the SNR around $15 \mathrm{~dB}$. The same happens to co-array-based MUSIC and EPRIT at SNR around $10 \mathrm{~dB}$. Nonetheless, there is still a considerable gap between the performance of the WLS estimator and co-array-based MUSIC and ESPRIT under this condition.

\section{CONCLUSION}

In this paper, a novel WLS estimator for the co-array-based DoA estimation is proposed. It is shown that the asymptotic performance of the proposed WLS estimator approaches the CRB when optimal weighting matrix is selected. This implies that the proposed WLS estimator is asymptotically statistically efficient. Simulation results illustrate the superior performance of the proposed WLS estimator compared to co-array-based MUSIC and ESPRIT in terms of estimation accuracy.

\section{ACKNOWLEDGMENT}

This work is supported by the National Research Fund, Luxembourg under AFR grant for bilateral Ph.D. project (Project Reference 11228830) on Compressive Sensing for Ranging and Detection in Automotive Applications.

\section{REFERENCES}

[1] S. S. Haykin, J. Litva, , and T. J. Shepherd, Eds., Radar Array Processing. Berlin, Germany: Springer-Verlag, 1993.

[2] P. Stoica and A. Nehorai, "Performance study of conditional and unconditional direction-of-arrival estimation," IEEE Transactions on Acoustics, Speech, and Signal Processing, vol. 38, no. 10, pp. 17831795, Oct 1990.

[3] A. Moffet, "Minimum-redundancy linear arrays," IEEE Transactions on Antennas and Propagation, vol. 16, no. 2, pp. 172-175, Mar 1968.

[4] P. P. Vaidyanathan and P. Pal, "Sparse sensing with co-prime samplers and arrays," IEEE Transactions on Signal Processing, vol. 59, no. 2, pp. 573-586, Feb 2011
[5] P. Pal and P. P. Vaidyanathan, "Nested arrays: A novel approach to array processing with enhanced degrees of freedom," IEEE Transactions on Signal Processing, vol. 58, no. 8, pp. 4167-4181, Aug 2010.

[6] C. L. Liu and P. Vaidyanathan, "Cramér-rao bounds for coprime and other sparse arrays, which find more sources than sensors," Digital Signal Processing, vol. 61, pp. 43 - 61, 2017.

[7] M. Wang and A. Nehorai, "Coarrays, music, and the cram ér-rao bound," IEEE Transactions on Signal Processing, vol. 65, no. 4, pp. 933-946, Feb 2017.

[8] J. Steinwandt, F. Roemer, and M. Haardt, "Performance analysis of esprit-type algorithms for co-array structures," in 2017 IEEE 7th International Workshop on Computational Advances in Multi-Sensor Adaptive Processing (CAMSAP), Dec 2017, pp. 1-5.

[9] C. L. Liu and P. P. Vaidyanathan, "Remarks on the spatial smoothing step in coarray music," IEEE Signal Processing Letters, vol. 22, no. 9, pp. 1438-1442, Sept 2015.

[10] Y. D. Zhang, M. G. Amin, and B. Himed, "Sparsity-based doa estimation using co-prime arrays," in 2013 IEEE International Conference on Acoustics, Speech and Signal Processing, May 2013, pp. 3967-3971.

[11] P. Pal and P. P. Vaidyanathan, "A grid-less approach to underdetermined direction of arrival estimation via low rank matrix denoising," IEEE Signal Processing Letters, vol. 21, no. 6, pp. 737-741, June 2014.

[12] Z. Tan and A. Nehorai, "Sparse direction of arrival estimation using co-prime arrays with off-grid targets," IEEE Signal Processing Letters, vol. 21, no. 1, pp. 26-29, Jan 2014.

[13] S. Sedighi, B. Shankar, and B. Ottersten, "Consistent least squares estimator for co-array-based doa estimation," in 10th IEEE Sensor Array and Multichannel Signal Processing Workshop (SAM), July 2018.

[14] _ _A statistically efficient weighted least squares estimator for coarray-based doa estimation," Submitted to IEEE Transactions on Signal Processing, 2018.

[15] P. Stoica and K. C. Sharman, "Novel eigenanalysis method for direction estimation," IEE Proceedings F - Radar and Signal Processing, vol. 137, no. 1, pp. 19-26, Feb 1990.

[16] B. Ottersten, M. Viberg, and T. Kailath, "Performance analysis of the total least squares esprit algorithm," IEEE Transactions on Signal Processing, vol. 39, no. 5, pp. 1122-1135, May 1991.

[17] A. Paulraj, B. Ottersten, R. Roy, A. Swindlehurst, G. Xu, and T. Kailath, "16 subspace methods for directions-of-arrival estimation," Handbook of Statistics, vol. 10, pp. 693-739, 1993.

[18] M. Jansson and B. Ottersten, "Structured covariance matrix estimation: a parametric approach," in 2000 IEEE International Conference on Acoustics, Speech, and Signal Processing. Proceedings (Cat. No.00CH37100), vol. 5, June 2000, pp. 3172-3175 vol.5. 